\title{
$\beta$-Adrenoceptor Blockade in the Basolateral Amygdala, But Not the Medial Prefrontal Cortex, Rescues the Immediate Extinction Deficit
}

\author{
Thomas F Giustino',2, Jocelyn R Seemann ${ }^{1,2}$, Gillian M Acca',2, Travis D Goode ${ }^{1,2}$, Paul J Fitzgerald' and \\ Stephen Maren*,1,2 \\ 'Department of Psychology, Texas A\&M University, College Station, TX, USA; ${ }^{2}$ Institute for Neuroscience, Texas A\&M University, College Station, \\ TX, USA
}

\begin{abstract}
Early psychological interventions, such as exposure therapy, rely on extinction learning to reduce the development of stress- and traumarelated disorders. However, recent research suggests that extinction often fails to reduce fear when administered soon after trauma. This immediate extinction deficit (IED) may be due to stress-induced dysregulation of neural circuits involved in extinction learning. We have shown that systemic $\beta$-adrenoceptor blockade with propranolol rescues the IED, but impairs delayed extinction. Here we sought to determine the neural locus of these effects. Rats underwent auditory fear conditioning and then received either immediate (30 min) or delayed $(24 \mathrm{~h})$ extinction training. We used bilateral intracranial infusions of propranolol into either the infralimbic division of the medial prefrontal cortex (mPFC) or the basolateral amygdala (BLA) to examine the effects of $\beta$-adrenoceptor blockade on immediate and delayed extinction learning. Interestingly, intra-BLA, but not intra-mPFC, propranolol rescued the IED; animals receiving intra-BLA propranolol prior to immediate extinction showed less spontaneous recovery of fear during extinction retrieval. Importantly, this was not due to impaired consolidation of the conditioning memory. In contrast, neither intra-BLA nor intra-mPFC propranolol affected delayed extinction learning. Overall, these data contribute to a growing literature suggesting dissociable roles for key nodes in the fear extinction circuit depending on the timing of extinction relative to conditioning. These data also suggest that heightened noradrenergic activity in the BLA underlies stress-induced extinction deficits. Propranolol may be a useful adjunct to behavioral therapeutic interventions in recently traumatized individuals who are at risk for developing trauma-related disorders.
\end{abstract}

Neuropsychopharmacology (2017) 42, 2537-2544; doi: I 0.1038/npp.20 I7.89; published online 3I May 2017

\section{INTRODUCTION}

Early interventions (eg, exposure therapy) after psychological trauma are aimed at reducing the development of stressorand trauma-related disorders such as post-traumatic stress disorder (PTSD). The timing of these therapeutic interventions relative to trauma may be a key factor in their longterm success. However, both human and animal research suggest that early interventions may actually worsen symptoms (Bryant, 2002; Maren and Chang, 2006) relative to delayed interventions. Using Pavlovian fear conditioning and extinction procedures in rats, we and others have similarly found that administering extinction trials soon (minutes to several hours) after conditioning yields little long-term extinction (Kim et al, 2010; Macpherson et al, 2013; Maren and Chang, 2006; Maren, 2014; Stafford et al, 2013). This 'immediate extinction deficit' (IED) may be

\footnotetext{
*Correspondence: Dr S Maren, Department of Psychology and Institute for Neuroscience, Texas A\&M University, TAMU 4235, College Station, TX 77843-4235, USA, Tel: 979 458-7960, E-mail: maren@tamu.edu Received 10 March 2017; revised 21 April 2017; accepted 24 April 2017; accepted article preview online 2 May 2017
}

caused by stress-induced impairments of extinction learning and recall (Maren and Holmes, 2016), because it is not observed with weak conditioning procedures (Maren and Chang, 2006). Thus, 'extinction-like' therapies in humans may fail when administered soon after trauma (when victims are still under duress) and lead to fear relapse (Bouton, 2000; Goode and Maren, 2014).

Considerable evidence indicates that stress-induced impairments in extinction learning are mediated by the medial prefrontal cortex (mPFC) (Holmes and Wellman, 2009; Maren and Holmes, 2016), a brain area that is critical for extinction learning (Giustino and Maren, 2015; Milad and Quirk, 2012). In particular, activity in the infralimbic (IL) subdivision of the mPFC is thought to underlie extinction learning (Bukalo et al, 2015; Do-Monte et al, 2015; Fitzgerald et al, 2014). One possibility is that stressinduced elevations in noradrenergic signaling dysregulate mPFC function and impair mPFC-dependent psychological processes, including extinction. Consistent with this possibility, it has long been appreciated that norepinephrine (NE) is chronically elevated in patients with PTSD (Geracioti et al, 2001; Southwick et al, 1999), and animal research has shown 
that stressors, including footshock, result in elevated prefrontal NE release (Hugues et al, 2007). NE may also indirectly influence the mPFC by modulating its inputs (Arnsten et al, 2015), including the basolateral amygdala (BLA), another brain area implicated in stress-impaired extinction learning (Maren and Holmes, 2016).

In support of this model, we have recently shown that systemic administration of D,L-propranolol, a non-selective $\beta$-adrenoceptor antagonist, immediately after fear conditioning (and just prior to immediate extinction) facilitates extinction retention and 'rescues' the IED (Fitzgerald et al, 2015). Propranolol also mitigates conditioning-induced suppression of IL spontaneous single-unit activity, which may have facilitated extinction learning under stress. In contrast, systemic propranolol administered prior to delayed extinction ( $24 \mathrm{~h}$ after conditioning) impaired extinction retention (Fitzgerald et al, 2015). Interestingly, recent work indicates that oral propranolol administration in humans also facilitates extinction learning under some conditions (Kroes et al, 2016). Little is known, however, concerning the brain areas mediating the effects of systemic propranolol on extinction learning. Indeed, there is some evidence that propranolol administered directly to the mPFC impairs delayed extinction (Mueller et al, 2008), but the brain regions mediating the effects of propranolol on immediate extinction are not known. Here we examine the contribution of $\beta$-adrenoceptor activity in the IL and BLA during immediate and delayed extinction using bilateral intracranial infusions of propranolol.

\section{MATERIALS AND METHODS}

\section{Subjects}

One hundred and twenty-eight experimentally naïve adult male Long-Evans rats (Blue-Spruce strain; weighing 200-224 g; 50-57 days old) were obtained from a commercial supplier (Envigo, Indianapolis, IN). The rats were individually housed in cages within a temperature- and humiditycontrolled vivarium, and kept on a 14:10 h light/dark cycle (lights on at 0700 hours) with ad libitum access to food and water. All experiments took place during the light phase of the cycle. Rats were handled for $\sim 30 \mathrm{~s}$ a day for 5 days to habituate them to the experimenter before any behavioral testing or surgical procedures were carried out. All experiments were conducted at Texas A\&M University with full approval from its Animal Care and Use Committee.

\section{Surgical Procedures}

Rats were randomly assigned to experimental groups prior to surgery. One week before behavioral testing took place, rats were anesthetized with isoflurane (5\% induction, $\sim 2 \%$ maintenance) and secured in a stereotaxic apparatus (Kopf Instruments, Tujunga, CA). The scalp was incised and retracted; three burr holes were drilled for anchor screws; additional holes were drilled in the skull overlying the mPFC or amygdala to allow for cannula implantation. The IL cortex was targeted by a single cannula $(8 \mathrm{~mm}, 26$ gauge; Plastics One) implanted on the midline (AP: +2.7, ML: +1.0 (insertion point), DV: -4.9 at an $11^{\circ}$ angle; all coordinates relative to bregma at skull surface). This procedure has been used by other laboratories to pharmacologically manipulate the IL in both hemispheres of the brain (Mueller et al, 2008). For the BLA, guide cannulae $(10 \mathrm{~mm}, 26$ gauge) were implanted bilaterally (AP: $-2.9, \mathrm{ML}: \pm 4.8$, DV: -8.55 ). Three jeweler's screws were affixed to the skull, and the skull surface was covered with dental cement to secure the cannulae to the skull. Stainless steel dummy cannulae (30 gauge) were inserted into the guide cannulae (extending $1 \mathrm{~mm}$ beyond the end of the guide). Rats were allowed to recover on a warmed heating pad prior to returning to the vivarium. Dummy cannulae were replaced twice in the week following surgery (prior to behavior) and again after the infusion session.

\section{Drug Infusions}

Intracranial infusions were made as previously described (Acca et al, 2017). Briefly, rats were transported to an infusion room (either from the conditioning room for immediate and no-extinction procedures or from the vivarium for delayed extinction). Dummies were then removed, and stainless steel injectors (33 gauge) connected to Hamilton syringes mounted in an infusion pump were inserted into the guide cannulae for intracranial infusions. All infusions were made approximately $20 \mathrm{~min}$ prior to the extinction or no-extinction session. D,L-Propranolol hydrochloride was obtained from a commercial supplier (SigmaAldrich) and was dissolved in sterile saline $(10 \mu \mathrm{g} / \mu \mathrm{l}$ for $\mathrm{mPFC}$ and $5 \mu \mathrm{g} / \mu \mathrm{l}$ for BLA). Infusions $(0.5 \mu \mathrm{l} /$ target $)$ were made at a rate of $0.25 \mu \mathrm{l} / \mathrm{min}$ for $2 \mathrm{~min}$ and the injectors were left in place for $1 \mathrm{~min}$ to allow for diffusion (mPFC: $5 \mu \mathrm{g}$ along midline; BLA: $2.5 \mu \mathrm{g} /$ hemisphere). The propranolol dose and volume were chosen based on previous reports (Debiec and Ledoux, 2004; Dębiec et al, 2011; Mueller et al, 2008). After the infusions, clean dummies were secured to the guide cannulae.

\section{Behavioral Apparatus and Procedures}

The behavioral procedures were conducted in 16 standard rodent conditioning chambers $(30 \times 24 \times 21 \mathrm{~cm}$; Med Associates, St Albans, VT) housed in sound-attenuating cabinets. Each chamber consisted of two aluminum sides, a Plexiglas rear wall and top, and a hinged Plexiglas door. The grid floor contained 19 stainless steel rods ( $4 \mathrm{~mm}$ diameter) spaced $1.5 \mathrm{~cm}$ apart (center-to-center). Rods were connected to a shock source and solid-state grid scrambler (Med Associates) for the delivery of footshocks. A loudspeaker mounted on the outside of a grating in one aluminum wall was used to deliver auditory stimuli. Locomotor activity was transduced into an electrical signal by a load cell under the floor of the chamber to automatically measure freezing.

Approximately 1 week after surgery, rats underwent fear conditioning, extinction (immediate, no-extinction, or delayed), and extinction retrieval sessions using an 'ABB' design: conditioning occurred in context $\mathrm{A}$ and extinction training and retrieval testing occurred in context $B$. Rats were run in squads of eight. For conditioning (context A), rats were transported from the vivarium to the behavioral room in black plastic transport boxes. The conditioning chambers were cleaned with a $1 \%$ ammonium hydroxide solution and a metal pan beneath the grid floor contained a thin layer of the 
a
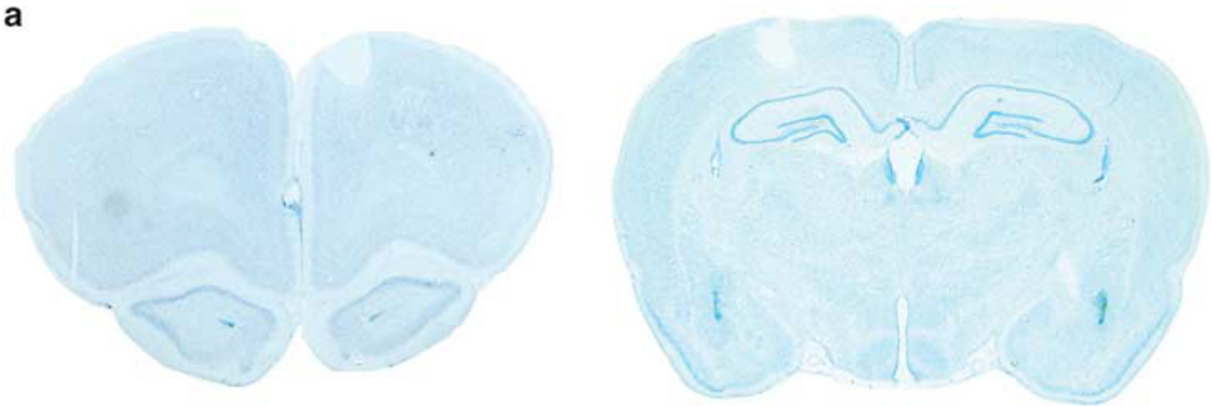

b

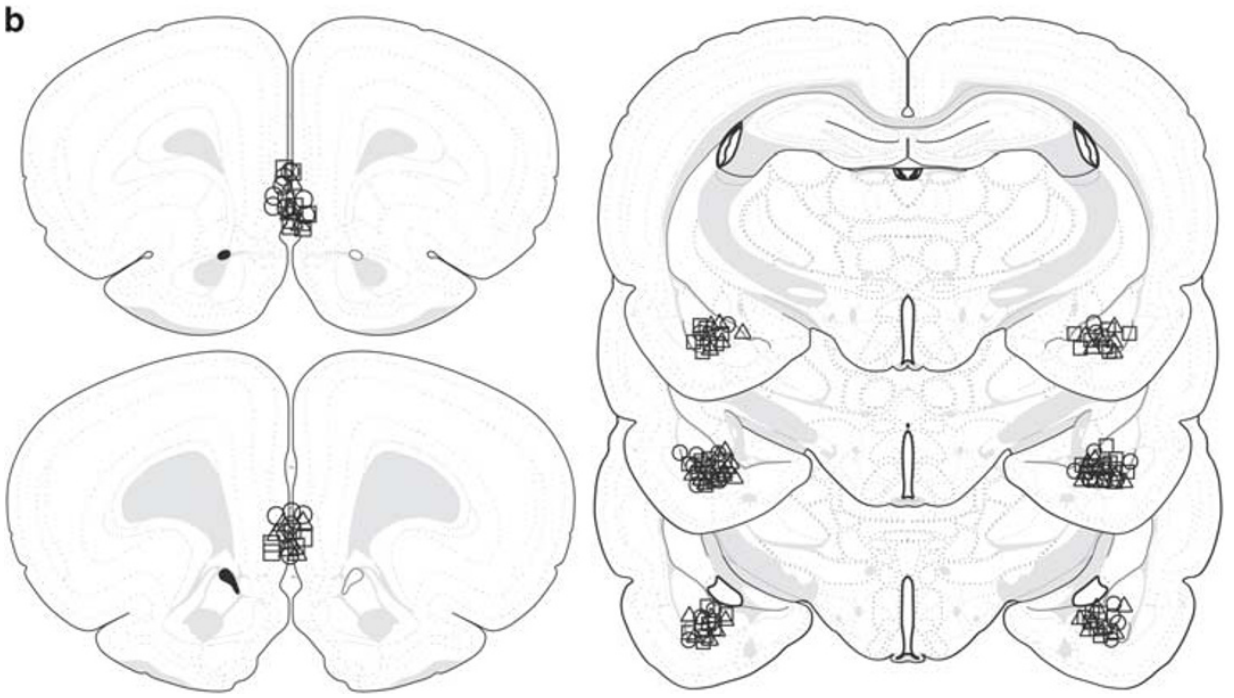

Figure I Histology. (a) Photomicrographs of representative thionin-stained coronal sections depicting cannula placements in the mPFC (left) or BLA (right). (b) Cannula placements for all the animals are depicted in schematic coronal sections. For ease of illustration, we plot placements across the three extinction manipulations (immediate extinction = triangles, no-extinction = squares, and delayed extinction = circles); the distribution of placements was similar across experiments and drug groups.

same solution. The room had red ambient lighting and the sound attenuating cabinet doors were closed prior to beginning the session. Conditioning consisted of a 3 -min stimulus-free baseline period followed by 5 tone $(10 \mathrm{~s}, 2 \mathrm{kHz}$, $80 \mathrm{~dB}$ )-shock ( $2 \mathrm{~s}, 1 \mathrm{~mA}$ ) pairings (shock onset occurred at tone offset) with a $1 \mathrm{~min}$ inter-trial interval (ITI) between each tone presentation. Rats remained in the chamber for 3 min following the last footshock.

For the extinction, no-extinction, and retrieval sessions (context B), the rats were transported in white plastic boxes. Chambers were cleaned with a $1 \%$ acetic acid solution and a metal pan beneath the grid floor contained a thin layer of the same solution. House lights and ventilation fans within each chamber were turned on and the room containing the chambers was illuminated with overhead white fluorescent lights. The doors of the sound attenuating cabinets were left open. After a 3-min baseline period, rats received 45 tonealone trials (1 min ITI) and remained in the chamber for 3 min following the last tone. The extinction training and retrieval test sessions were identical. Rats in the 'noextinction groups' underwent an identical procedure except that no tone-alone trials were delivered during the initial extinction session (ie, soon after conditioning and intra-cranial infusions).

In Experiment 1, we examined the influence of intracranial propranolol infusions on immediate extinction
(BLA VEH, $n=12$; mPFC VEH, $n=6$; BLA PROP, $n=14$; mPFC PROP, $n=5$ ). We also included a no-extinction control group (BLA VEH, $n=12$; $\mathrm{mPFC}$ VEH, $n=7$; BLA PROP, $n=14$; mPFC PROP, $n=6$ ) to determine whether intra-cranial propranolol affected consolidation of the conditioning memory (Fitzgerald et al, 2015). In Experiment 2 , we examined the effects of intra-cranial propranolol infusions on delayed extinction (BLA VEH, $n=6$; mPFC VEH, $n=7$; BLA PROP, $n=6$; mPFC PROP, $n=7)$. The immediate and no-extinction conditions for the BLA placements were conducted in replicate, which accounts for the greater number of subjects in those groups.

\section{Histology}

Rats were overdosed with sodium pentobarbital $(100 \mathrm{mg} / \mathrm{kg}$ ) and perfused transcardially with $0.9 \%$ saline followed by $10 \%$ formalin. Brains were extracted from the skull and post-fixed in a $10 \%$ formalin solution for $24 \mathrm{~h}$ followed by a $30 \%$ sucrose solution where they remained for a minimum of $48 \mathrm{~h}$. After the brains were fixed, coronal sections $(40 \mu \mathrm{m}$ thickness) were made on a cryostat $\left(-20^{\circ} \mathrm{C}\right)$, mounted on subbed microscope slides, and stained with thionin $(0.25 \%)$ to visualize cannula placements (Figure 1). Twenty-six rats with cannula placements that were not located within the target region were excluded from the analyses. 


\section{Data Analysis}

Data were analyzed with conventional parametric statistics (StatView; SAS Institute). Freezing for each trial was averaged across a 70-s interval, which included both the CS (10-s) and ITI (60-s). Freezing during the CS+ITI period is highly correlated with freezing to the CS itself, but is less susceptible to competition by the CS-elicited orienting response. Two-way analysis of variance (ANOVA) and repeated-measures ANOVA were used to assess general main effects and interactions $(\alpha=0.05)$. Results are shown as mean \pm SEM.

\section{RESULTS}

\section{Experiment 1: Intra-BLA Propranolol Rescues the IED}

This experiment sought to determine whether antagonizing $\beta$-adrenoceptors in the IL division of the mPFC or the BLA would facilitate extinction learning soon after conditioning. Rats were first conditioned with 5 tone-shock pairings in Context A. Immediately after conditioning, rats received either intra-mPFC or intra-BLA propranolol (or vehicle) followed $(\sim 20 \mathrm{~min})$ by extinction trials in a new context (Context B). Forty-eight hours later, the animals were returned to Context $\mathrm{B}$ for an extinction retrieval session. There were no differences in the behavior of control rats receiving $\mathrm{VEH}$ infusions into the IL or BLA, so these groups were combined to form a single control group.

As shown in Figure 2a, rats exhibited low levels of freezing behavior during the baseline period prior to the first conditioning trial, and increased their freezing behavior across conditioning trials; there were no differences between the groups (the animals were drug-free during conditioning). An ANOVA with a between-subjects variable of group and a within-subjects variable of trial revealed only a significant effect of trial $(\mathrm{F}(2,5)=32.9, p<0.01)$. During the extinction session (Figure 2b), intra-mPFC or intra-BLA propranolol infusions also did not affect freezing behavior. Rats in each group showed modest levels of freezing during the pre-CS baseline period, which is typical of recently shocked animals (Maren and Chang, 2006). Presentation of CS-alone trials augmented freezing in all of the animals, and this decreased similarly between groups over the course of extinction training. These impressions were confirmed in an ANOVA, which revealed only a main effect of trials $(F(2,5)=14.72$, $p<0.01$ ).

Forty-eight hours later the rats received a drug-free retention test consisting of a second extinction session (ie, 45 tone-alone trials in Context B). All groups showed low levels of baseline freezing (Figure 2c). CS presentations increased freezing in each group, and this decreased throughout the session (main effect of trials, $F(2,9)=10.06$, $p<0.01)$. Inspection of the data, however, suggests that BLA propranolol facilitated extinction retention in the earliest test trials. Indeed, an ANOVA with a between-subjects variable of group run on the first 5-trial block revealed a main effect of group (Figure 2c, $\mathrm{F}(2,34)=3.40, p<0.05$ ). Planned comparisons indicated that rats receiving intra-BLA, but not intra-mPFC, propranolol infusions prior to the immediate extinction session exhibited lower levels of freezing than rats in the other groups. In other words, noradrenergic antagonism in the BLA attenuated the IED and promoted long-term extinction retention. This is in agreement with an earlier report in which systemic propranolol facilitated extinction retention in the initial trials of the retrieval test (Fitzgerald et al, 2015).

Of course, it is possible that post-conditioning propranolol infusions into the BLA simply interfered with consolidation of the conditioning memory. To examine this possibility, another group of animals underwent a protocol identical to that described above except that extinction trials were not delivered after drug infusion (ie, 'no-extinction'; context exposure only). As expected, all groups displayed similar increases in freezing behavior across conditioning. An ANOVA revealed only a main effect of trials (Figure $2 \mathrm{~d}$, $\mathrm{F}(2,5)=45.43, p<0.01)$. During the context exposure session, all groups exhibited modest levels of freezing that decreased throughout the session. These observations were confirmed by an ANOVA, which revealed only a main effect of trials (Figure 2e, $\mathrm{F}(2,5)=7.8, p<0.01$ ). Forty-eight hours later, rats received a retrieval session consisting of $45 \mathrm{CS}$ alone trials. After the baseline period, all groups showed high levels of freezing to the CS, which decreased throughout the session at a similar rate. These observations were confirmed by ANOVA, which revealed only a main effect of trials (Figure 2f, $\mathrm{F}(2,9)=17.4, p<0.01$ ). Hence, post-conditioning propranolol in the absence of extinction training did not affect the consolidation of fear (Debiec and Ledoux, 2004; Fitzgerald et al, 2015; Schiff et al, 2017).

\section{Experiment 2: Intra-BLA or Intra-mPFC Propranolol Does Not Affect Delayed Extinction}

Previous experiments have revealed that systemic (Fitzgerald et al, 2015) or intra-mPFC (Mueller et al, 2008) propranolol impairs extinction using a standard delayed protocol. Here we sought to compare the effects of intra-BLA and intramPFC propranolol using a delayed extinction procedure. Animals underwent a behavioral protocol similar to that in Experiment 1 except that drug infusion (and extinction) occurred $24 \mathrm{~h}$ after fear conditioning. Similar to the experiments above, all groups exhibited low levels of freezing during the pre-conditioning baseline period and increased freezing over the course of conditioning. This was confirmed by an ANOVA, which revealed only a main effect of trials (Figure 3a, $\mathrm{F}(2,5)=37.34, p<0.01$ ). Twenty-four hours after conditioning, the rats received intracranial infusions prior to delayed extinction training. Intra-cranial propranolol administration did not affect freezing during the extinction session (Figure $3 \mathrm{~b}, \mathrm{~F}(2,5)=28.94, p<0.01$ ), nor did it affect the retention of extinction the following day (Figure $3 \mathrm{c}$ ). A repeated measures ANOVA revealed no effect of drug on extinction performance; all groups showed a small increase in CS-elicited freezing across the test session (Figure $3 c$, main effect of trials, $\mathrm{F}(2,90)=7.85, p<0.01)$.

To compare the behavioral outcomes across the experiments, we analyzed the first 5-trial block of the extinction retrieval test for each experimental condition. As shown in Figure 4, rats that received $\mathrm{VEH}$ or propranolol infusions into the MPFC exhibited an IED; they exhibited freezing that was no different from non-extinguished controls (and substantially higher than that after delayed extinction). In contrast, rats that received intra-BLA propranolol infusions 

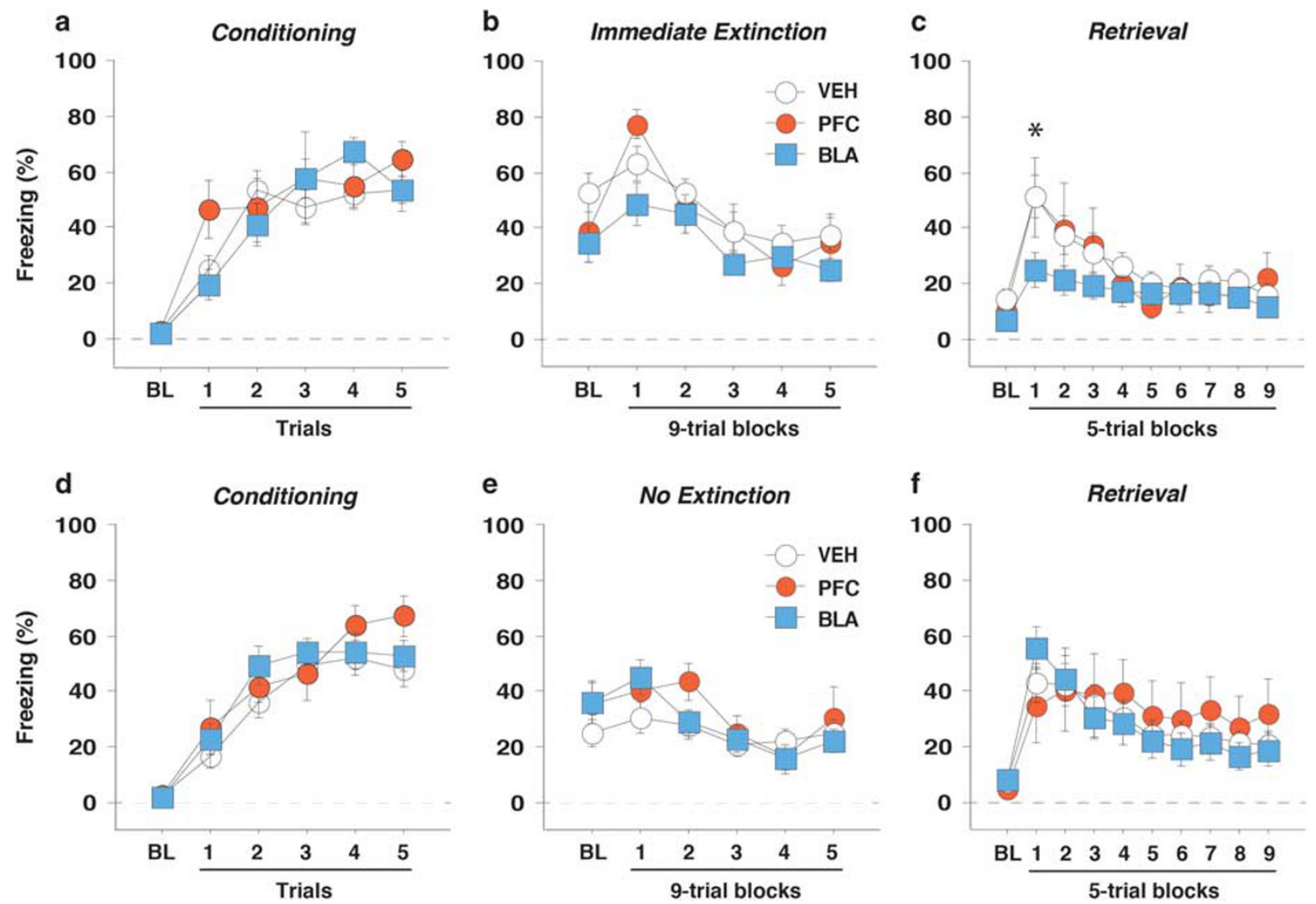

Figure 2 Intra-BLA propranolol rescues the immediate extinction deficit. (a) Percentage of freezing behavior (means \pm SEMs) during fear conditioning in animals that underwent immediate extinction (VEH, $n=18$; mPFC, $n=5 ; B L A n=14$ ). Intra-cranial injections were made immediately after fear conditioning. (b) Percentage of freezing behavior (means \pm SEMs) during the extinction session, which was conducted 20 min after fear conditioning. Intra-cranial infusions of propranolol into the BLA or PFC did not affect freezing behavior during the extinction session. (c) Percentage of freezing behavior (means \pm SEMs) during a second drug-free extinction session, which served as an index of extinction retention. Rats that received intra-BLA propranolol immediately after fear conditioning exhibited lower levels of freezing than those receiving $\mathrm{MPFC}$ propranolol or vehicle infusions. This suggests that intra-BLA propranolol facilitated extinction retention. ${ }^{*} p<0.05$. (d) Percentage of freezing behavior (means \pm SEMs) during fear conditioning in control animals that received post-training intracranial drug injections, but did not undergo extinction (VEH, $n=19 ; \mathrm{mPFC}, n=6$; BLA $n=14$ ). Intra-cranial injections were made immediately after fear conditioning. (e) Percentage of freezing behavior (means \pm SEMs) during the context exposure session, which was conducted 20 min after fear conditioning; tones were not delivered during this session. Intra-cranial infusions of propranolol into the BLA or mPFC did not affect freezing behavior during the exposure session. (f) Percentage of freezing behavior (means \pm SEMs) during a drug-free extinction session, which served as an index of the retention of conditioned fear. Post-conditioning intra-cranial propranolol infusions did not affect freezing behavior during the retention test; propranolol facilitated extinction retention (c) rather than disrupting the consolidation of the conditioning memory.
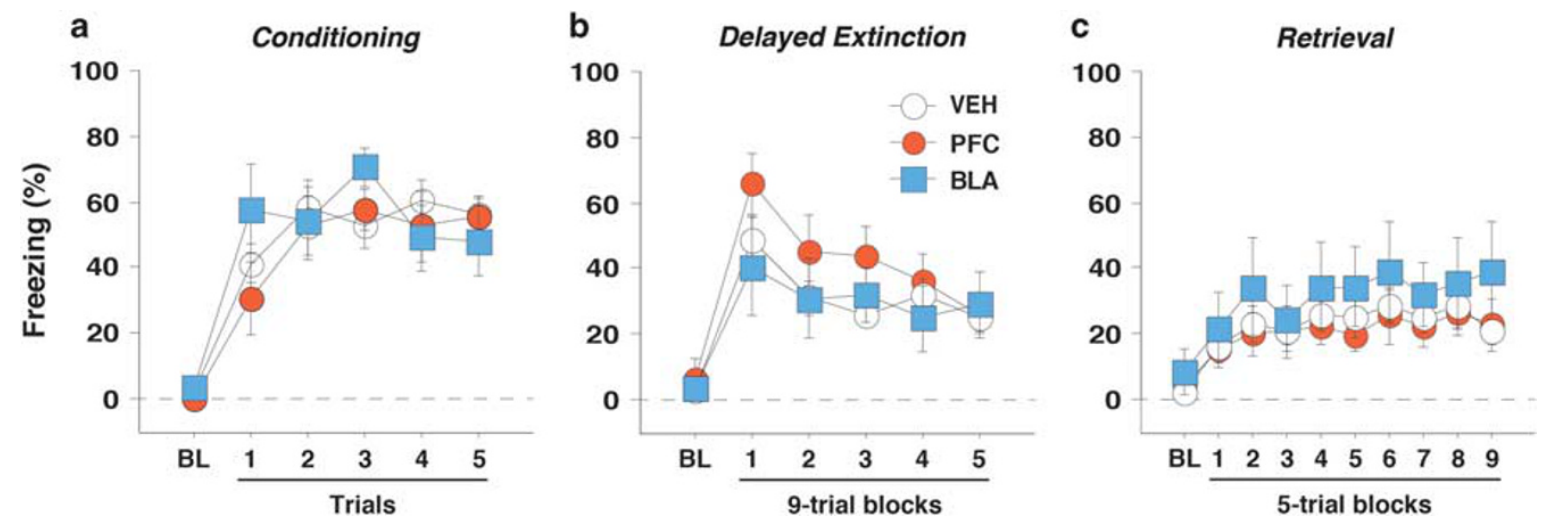

Figure 3 Intra-BLA or intra-mPFC propranolol does not affect delayed extinction. (a) Percentage of freezing behavior (means \pm SEMs) during fear conditioning (VEH $n=13, \operatorname{mPFC} n=7, \mathrm{BLA} n=6)$. (b) Percentage of freezing behavior (means \pm SEMs) during a delayed extinction session that occurred $24 \mathrm{~h}$ after conditioning (and immediately after intra-cranial infusions). Drug infusion did not alter freezing behavior between groups. (c) Percentage of freezing behavior (means \pm SEMs) during a drug-free extinction retrieval test that took place $24 \mathrm{~h}$ following extinction. Prior drug did not impact extinction retrieval. 


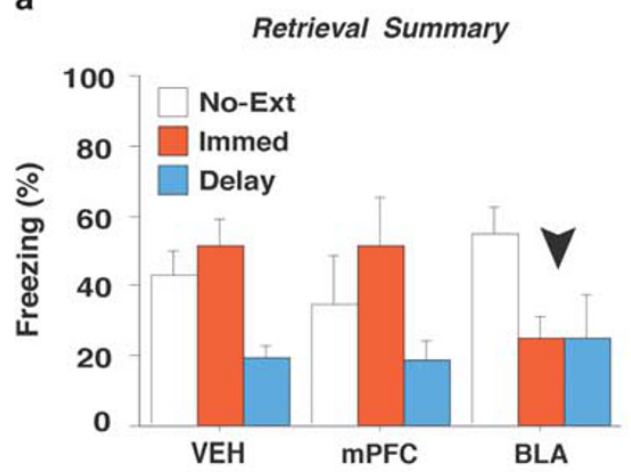

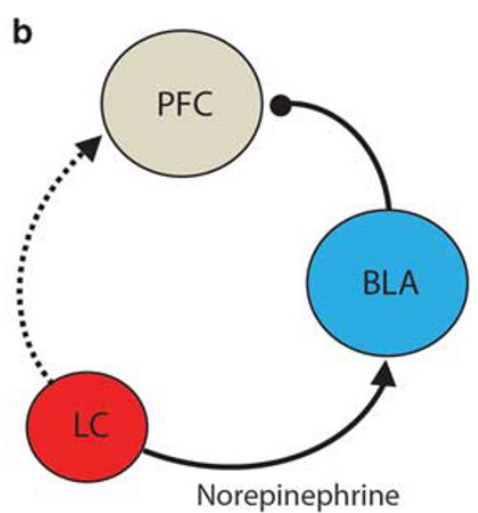

Figure 4 Summary and circuit model. (a) Percentage of freezing behavior (means \pm SEMs) plotting the average freezing for the first five trials (CS+ITI) during the retrieval test for all groups from each behavioral condition. Vehicle rats showed high freezing, characteristic of the immediate extinction deficit, which did not differ from no-extinction controls. Rats that underwent immediate extinction following intra-BLA propranolol did not exhibit the IED, showing similarly low levels of freezing to animals that underwent delayed extinction. (b) We propose a circuit underlying the IED where locus coeruleus (LC) norepinephrine preferentially increases BLA activity, which ultimately dampens mPFC output resulting in impaired extinction.

did not exhibit an IED, and showed low levels of freezing under both extinction conditions. These impressions were confirmed in an ANOVA with variables of group and extinction condition, which revealed a main effect of extinction condition $(\mathrm{F}(2,2)=5.54, p<0.01)$. This supports the idea that the timing of extinction relative to conditioning is a key factor determining the long-term retention of extinction (ie, immediate extinction is impaired relative to delayed). In addition, we observed a significant group $\times$ extinction condition interaction $(\mathrm{F}(2,94)=2.59, p<0.05)$, which reveals the differential effect of intra-BLA propranolol on freezing in the IED relative to the other groups. Interestingly, we did not find that intra-mPFC propranolol impairs delayed extinction as has been previously reported (Mueller et al, 2008).

\section{DISCUSSION}

Fear extinction deficits, including the IED, are thought to reflect impaired mPFC function (Chang et al, 2010; Fitzgerald et al, 2015; Fucich et al, 2016; Giustino et al, 2016; Kim et al, 2010; Maren, 2014) and this may relate to elevated noradrenergic signaling (Fitzgerald et al, 2015; Giustino et al, 2016). We demonstrate here that selectively blocking $\beta$-adrenoceptors within the BLA enables extinction where it normally fails. This effect was not observed when propranolol was infused into the mPFC. Importantly, neither intra-mPFC nor intra-BLA propranolol altered fear memory consolidation or delayed extinction learning. These data suggest that heightened noradrenergic signaling in the BLA may be a particularly important component underlying stress-induced extinction deficits.

We and others have demonstrated that extinction learning is impaired when administered soon after conditioning (Hollis et al, 2016; Kim et al, 2010; Macpherson et al, 2013; Maren and Chang, 2006; Maren, 2014; Merz et al, 2016). The IL is thought to underlie successful extinction learning (Bukalo et al, 2015; Do-Monte et al, 2015). Therefore, it has been suggested that aberrant $\mathrm{mPFC}$ activity may result in extinction deficits (Fucich et al, 2016; Giustino and Maren, 2015; Maren, 2014; Milad and Quirk, 2012). Because immediate extinction takes place soon after conditioning, during a state of high psychological stress, we hypothesized that elevated NE (presumably released from the locus coeruleus) may subserve extinction deficits. Previous work suggests that NE levels are elevated during conditioning and delayed extinction in the MPFC and BLA (Galvez et al, 1996; Hugues et al, 2007; Ishizuka et al, 2000). We have recently shown that systemic propranolol rescues the IED and that this seemed to be due to stabilizing prefrontal activity soon after conditioning (Fitzgerald et al, 2015).

Importantly, the effects of systemic propranolol on the IED do not appear to be due to antagonism of $\beta$-adrenoceptors in the mPFC. We show that intra-mPFC propranolol has no effect on the three conditions tested: immediate extinction, fear memory consolidation, and delayed extinction. During delayed extinction, animals that received intra-mPFC propranolol tended to show moderately elevated levels of freezing; however, this was not significantly different from vehicle controls or rats that received intraBLA propranolol prior to delayed extinction. Our results contrast with others who have observed that intra-mPFC propranolol impairs delayed extinction learning (Mueller et al, 2008). However, there are several reasons that might explain this disparity. First, Mueller et al (2008) trained rats to lever press for food in the conditioning chambers, and then measured freezing coincident with response suppression during the CS. Response suppression might engage mPFC to a greater extent than the standard fear conditioning procedure used here. Second, Mueller et al (2008) used much shorter and weaker footshocks than those used in the present study. It is conceivable that these procedures resulted in relatively lower levels of fear-induced NE release during extinction, thereby rendering it more sensitive to intramPFC propranolol. Lastly, it is also possible that strain differences in the contribution of the mPFC to extinction learning accounted for the disparity in these reports (Chang and Maren, 2010).

An important finding in the present experiment is that post-conditioning propranolol alone did not affect the retention of fear conditioning. These data are in agreement with our systemic propranolol findings (Fitzgerald et al, 2015) and 
reveal that propranolol did not attenuate the IED by impairing the consolidation of the conditioning memory. This is in line with work that has demonstrated that $\beta$-adrenoceptor activity is critical for the acquisition, but not consolidation, of conditioned fear (Bush et al, 2010; Debiec and Ledoux, 2004; Roozendaal et al, 2006; Schiff et al, 2017). Here, we confirm and extend these results. Our data suggest that while elevated noradrenergic activity may not be directly involved in the consolidation of the CS-US memory, heightened noradrenergic activity soon after conditioning appears to interfere with the successful acquisition and retention of a new and competing extinction memory. Indeed, the IED may be due to a deficit in the consolidation of the extinction memory insofar as within-session extinction proceeds normally during immediate extinction and is unaffected by intra-BLA propranolol infusions. Nonetheless, systemic propranolol influences prefrontal cortical neuronal activity soon after fear conditioning, and this might influence encoding of long-term extinction memories.

Interestingly, the present results reveal that $\beta$-adrenoceptors in the BLA mediate the effects of systemic propranolol on the IED and possibly shock-induced changes in mPFC spike firing (Fitzgerald et al, 2015). While our previous work suggested that the mPFC may be a key locus of action for propranolol, it is conceivable that shock-induced changes in mPFC activity are regulated by $\mathrm{mPFC}$ afferents (including the BLA). Indeed, heightened $\beta$-adrenoceptor activation promotes BLA excitability during conditioning (Skelly et al, 2017). This may modulate mPFC activity (Arnsten, 2009; Arnsten et al, 2015; Ji et al, 2010), leading to the IED. Consistent with this idea, Lin et al (2016) showed that extinction deficits (caused by single-prolonged stress) are associated with increased $\mathrm{NE}$ in both the $\mathrm{mPFC}$ and amygdala. In addition, it has recently been shown that propranolol infusions in the BLA facilitate the induction of hippocampal-prefrontal synaptic plasticity (Lim et al, 2017), which has previously been implicated in extinction learning (Deschaux et al, 2011; Peters et al, 2010; Stafford et al, 2012). Ultimately, the BLA is well positioned to modulate the contribution of the mPFC to extinction learning.

Importantly, our data show that intra-BLA propranolol prior to delayed extinction had no effect on extinction retrieval. This time-dependent enhancement of extinction suggests NE levels may be beyond 'optimal' soon after conditioning, impairing prefrontal processing and resulting in the IED. However, stress (and NE) may be relatively lower at the onset of delayed extinction, leading to decreased recruitment of low-affinity $\beta$-adrenoceptors in either the $\mathrm{mPFC}$ or BLA. Considerable data reveal that stress increases the activity of locus coeruleus (LC) neurons (Bangasser et al, 2016; Valentino and Van Bockstaele, 2008), and this is associated with increased noradrenergic release in the BLA and $\mathrm{mPFC}$, which may play a role in learning and memory (Berridge and Waterhouse, 2003; Sara, 2015; Uematsu et al, 2015). We propose that LC-driven increases in BLA excitability through activation of $\beta$-adrenoceptors might ultimately suppress mPFC activity, thereby undermining successful extinction when it occurs soon after conditioning (Figure 4b). Indeed, it is possible that different populations of BLA- and $\mathrm{mPFC}$-projecting neurons in the LC are engaged during immediate and delayed extinction.
Overall, the present data contribute to a growing literature suggesting dissociable roles for key nodes in the fear extinction circuit depending on the timing of extinction relative to conditioning. Our data suggest that elevated noradrenergic activity in the BLA, but not the $\mathrm{mPFC}$, underlies extinction deficits during high psychological stress. Further work is required to explore this circuitry, but one possibility is that LC-NE enhances BLA excitability which ultimately suppresses mPFC activity, resulting in the IED. Propranolol may therefore be a useful adjunct to behavioral therapeutic interventions in recently traumatized individuals who are at risk for developing trauma-related disorders (Giustino et al, 2016; Kroes et al, 2016).

\section{FUNDING AND DISCLOSURE}

This work was supported by grants from the National Institutes of Health (R01MH065961 to SM and F31MH107113 to TDG). SM was also supported by a McKnight Memory and Cognitive Disorders Award. The authors declare no conflict of interest.

\section{REFERENCES}

Acca GM, Mathew AS, Jin J, Maren S, Nagaya N (2017). Allopregnanolone induces state-dependent fear via the bed nucleus of the stria terminalis. Horm Behav 89: 137-144.

Arnsten AFT (2009). Stress signalling pathways that impair prefrontal cortex structure and function. Nat Rev Neurosci 10: 410-422.

Arnsten AFT, Raskind MA, Taylor FB, Connor DF (2015). The effects of stress exposure on prefrontal cortex: translating basic research into successful treatments for post-traumatic stress disorder. Neurobiol Stress 1: 89-99.

Bangasser DA, Wiersielis KR, Khantsis S (2016). Sex differences in the locus coeruleus-norepinephrine system and its regulation by stress. Brain Res 1641: 177-188.

Berridge CW, Waterhouse BD (2003). The locus coeruleusnoradrenergic system: modulation of behavioral state and statedependent cognitive processes. Brain Res Brain Res Rev 42: 33-84.

Bouton ME (2000). A learning theory perspective on lapse, relapse, and the maintenance of behavior change. Health Psychol 19: 57-63.

Bryant RA (2002). Early interventions following psychological trauma. CNS Spectr 7: 650-654.

Bukalo O, Pinard CR, Silverstein S, Brehm C, Hartley ND, Whittle $\mathrm{N}$ et al (2015). Prefrontal inputs to the amygdala instruct fear extinction memory formation. Sci Adv 1: 5 .

Bush DEA, Caparosa EM, Gekker A, Ledoux J (2010). Betaadrenergic receptors in the lateral nucleus of the amygdala contribute to the acquisition but not the consolidation of auditory fear conditioning. Front Behav Neurosci 4: 154.

Chang C, Berke JD, Maren S (2010). Single-unit activity in the medial prefrontal cortex during immediate and delayed extinction of fear in rats. PLoS ONE 5: e11971.

Chang C, Maren S (2010). Strain difference in the effect of infralimbic cortex lesions on fear extinction in rats. Behav Neurosci 124: 391-397.

Dębiec J, Bush DEA, LeDoux JE (2011). Noradrenergic enhancement of reconsolidation in the amygdala impairs extinction of conditioned fear in rats-a possible mechanism for the persistence of traumatic memories in PTSD. Depress Anxiety 28: 186-193. 
Debiec J, Ledoux JE (2004). Disruption of reconsolidation but not consolidation of auditory fear conditioning by noradrenergic blockade in the amygdala. Neuroscience 129: 267-272.

Deschaux O, Motanis H, Spennato G, Moreau J-L, Garcia R (2011). Reemergence of extinguished auditory-cued conditioned fear following a sub-conditioning procedure: effects of hippocampal and prefrontal tetanic stimulations. Neurobiol Learn Mem 95: 510-518.

Do-Monte FH, Manzano-Nieves G, Quiñones-Laracuente K, Ramos-Medina L, Quirk GJ (2015). Revisiting the role of infralimbic cortex in fear extinction with optogenetics. J Neurosci 35: 3607-3615.

Fitzgerald PJ, Giustino TF, Seemann JR, Maren S (2015). Noradrenergic blockade stabilizes prefrontal activity and enables fear extinction under stress. Proc Natl Acad Sci USA 112: E3729-37.

Fitzgerald PJ, Whittle N, Flynn SM, Graybeal C, Pinard CR, Gunduz-Cinar $\mathrm{O}$ et al (2014). Prefrontal single-unit firing associated with deficient extinction in mice. Neurobiol Learn Mem 113: 69-81.

Fucich EA, Paredes D, Morilak DA (2016). Therapeutic effects of extinction learning as a model of exposure therapy in rats. Neuropsychopharmacology 41: 3092-3102.

Galvez R, Mesches MH, McGaugh JL (1996). Norepinephrine release in the amygdala in response to footshock stimulation. Neurobiol Learn Mem 66: 253-257.

Geracioti TD, Baker DG, Ekhator NN, West SA, Hill KK, Bruce AB et al (2001). CSF norepinephrine concentrations in posttraumatic stress disorder. Am J Psychiatry 158: 1227-1230.

Giustino TF, Fitzgerald PJ, Maren S (2016). Revisiting propranolol and PTSD: memory erasure or extinction enhancement? Neurobiol Learn Mem 130: 26-33.

Giustino TF, Maren S (2015). The role of the medial prefrontal cortex in the conditioning and extinction of fear. Front Behav Neurosci 9: 298.

Goode TD, Maren S (2014). Animal models of fear relapse. ILAR J 55: 246-258.

Hollis F, Sevelinges Y, Grosse J, Zanoletti O, Sandi C (2016). Involvement of CRFR1 in the basolateral amygdala in the immediate fear extinction deficit. eNeuro 3: 5.

Holmes A, Wellman CL (2009). Stress-induced prefrontal reorganization and executive dysfunction in rodents. Neurosci Biobehav Rev 33: 773-783.

Hugues S, Garcia R, Léna I (2007). Time course of extracellular catecholamine and glutamate levels in the rat medial prefrontal cortex during and after extinction of conditioned fear. Synapse 61: 933-937.

Ishizuka Y, Ishida Y, Jin Q, Kato K, Kunitake T, Mitsuyama Y et al (2000). Differential profiles of nitric oxide and norepinephrine releases in the paraventricular nucleus region in response to mild footshock in rats. Brain Res 862: 17-25.

Ji G, Sun H, Fu Y, Li Z, Pais-Vieira M, Galhardo V et al (2010). Cognitive impairment in pain through amygdala-driven prefrontal cortical deactivation. J Neurosci 30: 5451-5464.

Kim SC, Jo YS, Kim IH, Kim H, Choi J-S (2010). Lack of medial prefrontal cortex activation underlies the immediate extinction deficit. J Neurosci 30: 832-837.

Kroes MCW, Tona K-D, den Ouden HEM, Vogel S, van Wingen GA, Fernández G (2016). How administration of the beta-blocker propranolol before extinction can prevent the return of fear. Neuropsychopharmacology 41: 1569-1578.

Lim EP, Dawe GS, Jay TM (2017). Activation of beta- and alpha-2adrenoceptors in the basolateral amygdala has opposing effects on hippocampal-prefrontal long-term potentiation. Neurobiol Learn Mem 137: 163-170.

Lin C-C, Tung C-S, Lin P-H, Huang C-L, Liu Y-P (2016). Traumatic stress causes distinctive effects on fear circuit catecholamines and the fear extinction profile in a rodent model of posttraumatic stress disorder. Eur Neuropsychopharmacol 26: 1484-1495.

Macpherson K, Whittle N, Camp M, Gunduz-Cinar O, Singewald $\mathrm{N}$, Holmes A (2013). Temporal factors in the extinction of fear in inbred mouse strains differing in extinction efficacy. Biol Mood Anxiety Disord 3: 13.

Maren S (2014). Nature and causes of the immediate extinction deficit: a brief review. Neurobiol Learn Mem 113: 19-24.

Maren S, Chang C (2006). Recent fear is resistant to extinction. Proc Natl Acad Sci USA 103: 18020-18025.

Maren S, Holmes A (2016). Stress and fear extinction. Neuropsychopharmacology 41: 58-79.

Merz CJ, Hamacher-Dang TC, Wolf OT (2016). Immediate extinction promotes the return of fear. Neurobiol Learn Mem 131: 109-116.

Milad MR, Quirk GJ (2012). Fear extinction as a model for translational neuroscience: ten years of progress. Annu Rev Psychol 63: 129-151.

Mueller D, Porter JT, Quirk GJ (2008). Noradrenergic signaling in infralimbic cortex increases cell excitability and strengthens memory for fear extinction. J Neurosci 28: 369-375.

Peters J, Dieppa-Perea LM, Melendez LM, Quirk GJ (2010). Induction of fear extinction with hippocampalinfralimbic BDNF. Science 328: 1288-1290.

Roozendaal B, Okuda S, Van der Zee EA, McGaugh JL (2006). Glucocorticoid enhancement of memory requires arousalinduced noradrenergic activation in the basolateral amygdala. Proc Natl Acad Sci USA 103: 6741-6746.

Sara SJ (2015). Locus Coeruleus in time with the making of memories. Curr Opin Neurobiol 35: 87-94.

Schiff HC, Johansen JP, Hou M, Bush DEA, Smith EK, Klein JE et al (2017). $\beta$-Adrenergic receptors regulate the acquisition and consolidation phases of aversive memory formation through distinct, temporally regulated signaling pathways. Neuropsychopharmacology 42: 895-903.

Skelly MJ, Ariwodola OJ, Weiner JL (2017). Fear conditioning selectively disrupts noradrenergic facilitation of GABAergic inhibition in the basolateral amygdala. Neuropharmacology 113: 231-240.

Southwick SM, Bremner JD, Rasmusson A, Morgan CA, Arnsten A, Charney DS (1999). Role of norepinephrine in the pathophysiology and treatment of posttraumatic stress disorder. Biol Psychiatry 46: 1192-1204.

Stafford JM, Maughan DK, Ilioi EC, Lattal KM (2013). Exposure to a fearful context during periods of memory plasticity impairs extinction via hyperactivation of frontal-amygdalar circuits. Learn Mem 20: 156-163.

Stafford JM, Raybuck JD, Ryabinin AE, Lattal KM (2012). Increasing histone acetylation in the hippocampus-infralimbic network enhances fear extinction. Biol Psychiatry 72: 25-33.

Uematsu A, Tan BZ, Johansen JP (2015). Projection specificity in heterogeneous locus coeruleus cell populations: implications for learning and memory. Learn Mem 22: 444-451.

Valentino RJ, Van Bockstaele E (2008). Convergent regulation of locus coeruleus activity as an adaptive response to stress. Eur J Pharmacol 583: 194-203. 